\title{
On the Boundedness of the Fractional Bergman Operators
}

\author{
Benoît F. Sehba \\ Department of Mathematics, University of Ghana, P.O. Box LG 62, Legon, Accra, Ghana \\ Correspondence should be addressed to Benoît F. Sehba; bsehba@gmail.com \\ Received 4 March 2017; Accepted 18 April 2017; Published 10 May 2017 \\ Academic Editor: Feyzi Başar \\ Copyright (C) 2017 Benoît F. Sehba. This is an open access article distributed under the Creative Commons Attribution License, \\ which permits unrestricted use, distribution, and reproduction in any medium, provided the original work is properly cited.
}

We give a necessary and sufficient condition for the boundedness of the Bergman fractional operators.

\section{Introduction and Statement of the Result}

We are interested in this note in the boundedness of the Bergman fractional operator. The Bergman fractional operator has been shown recently to be quite useful in understanding off-diagonal questions for the Bergman operator (see $[1,2]$ ). Our aim here is to provide a necessary and sufficient condition for the boundedness of this operator. In the next lines, we provide some notions and definitions needed in the sequel.

Let $\mathscr{H}$ be the upper-half plane, that is, the set $\{z=x+$ iy $\in \mathbb{C}: y>0\}$. We denote by $L_{\alpha}^{p}(\mathscr{H})$ the Lebesgue space $L^{p}\left(\mathscr{H}, y^{\alpha} d x d y\right)$, that is, the space of all functions $f$ such that

$$
\|f\|_{p, \alpha}^{p}:=\int_{\mathscr{H}}|f(x+i y)|^{p} y^{\alpha} d x d y<\infty .
$$

For $\alpha>-1$ and $1<p<\infty$, the weighted Bergman space $A_{\alpha}^{p}(\mathscr{H})$ is the subspace of $L_{\alpha}^{p}(\mathscr{H})$ consisting of analytic functions. It is well known that the Bergman space $A_{\alpha}^{2}(\mathscr{H})$ $(-1<\alpha<\infty)$ is a reproducing kernel Hilbert space with kernel $K_{z}^{\alpha}(w)=K^{\alpha}(z, w)=1 /(z-\bar{w})^{2+\alpha}$. That is, for any $f \in A_{\alpha}^{2}(\mathscr{H})$, the following representation holds:

$$
\begin{aligned}
f(w) & =P_{\alpha} f(w)=\left\langle f, K_{w}^{\alpha}\right\rangle_{\alpha} \\
& =\int_{\mathscr{H}} f(z) K^{\alpha}(w, z) d V_{\alpha}(z),
\end{aligned}
$$

where, for simplicity, we write $d V_{\alpha}(x+i y)=y^{\alpha} d x d y$. The positive Bergman operator $P_{\alpha}^{+}$is defined by

$$
P_{\alpha}^{+} f(w)=\int_{\mathscr{H}} f(z)\left|K^{\alpha}(w, z)\right| d V_{\alpha}(z) .
$$

Note that the boundedness of $P_{\alpha}^{+}$implies the boundedness of $P_{\alpha}$. It is an elementary exercise to prove that the Bergman projection $P_{\alpha}$ is bounded on $L_{\alpha}^{p}(\mathscr{H})$ if and only if $1<p<\infty$ (see, e.g., [3]).

The fractional Bergman operator $\mathscr{P}_{\alpha, \gamma}$ is defined by

$$
\mathscr{P}_{\alpha, \gamma} f(w)=\int_{\mathscr{H}} \frac{f(z)}{(w-\bar{z})^{2+\alpha-\gamma}} d V_{\alpha}(z),
$$

where $0 \leq \gamma<2+\alpha$. The corresponding positive operator will be denoted by $\mathscr{P}_{\alpha, \gamma}^{+}$and can be seen as the upper-half space analogue of the Riesz potential also known as fractional operator (see [4]). Note also that, for $\gamma=0, \mathscr{P}_{\alpha, \gamma}$ is just the Bergman projection.

We have the following necessary and sufficient condition for the boundedness of $\mathscr{P}_{\alpha, \gamma}$ and $\mathscr{P}_{\alpha, \gamma}^{+}$.

Theorem 1. Let $\alpha>-1,0 \leq \gamma<2+\alpha$, and $1<p \leq q<\infty$. Then the following conditions are equivalent:

(a) The operator $\mathscr{P}_{\alpha, \gamma}$ is bounded from $L^{p}\left(\mathscr{H}, d V_{\alpha}\right)$ to $L^{q}\left(\mathscr{H}, d V_{\alpha}\right)$

(b) The operator $\mathscr{P}_{\alpha, \gamma}^{+}$is bounded from $L^{p}\left(\mathscr{H}, d V_{\alpha}\right)$ to $L^{q}\left(\mathscr{H}, d V_{\alpha}\right)$.

(c) The following relation holds:

$$
\frac{1}{p}-\frac{1}{q}=\frac{\gamma}{2+\alpha}
$$

Unlike the case of the unit ball (see [5]), the above result can not be deduced from the boundedness of the families of Bergman-type operators considered in $[2,6]$. 
We remark that the boundedness of the operator $\mathscr{P}_{\alpha, \gamma}^{+}$ from $L^{p}\left(\mathscr{H}, d V_{\alpha}\right)$ to $L^{q}\left(\mathscr{H}, d V_{\alpha}\right)$ implies the boundedness of $P_{\alpha}^{+}$from $L^{p}\left(\mathscr{H}, d V_{\alpha}\right)$ to $L^{q}\left(\mathscr{H}, d V_{\beta}\right)$, where $\beta=q \gamma+\alpha$. It follows that we have the following.

Proposition 2. Let $\alpha>-1,0 \leq \gamma<2+\alpha$, and $1<p \leq q<\infty$. Then the following conditions are equivalent:

(a) The operator $P_{\alpha}$ is bounded from $L^{p}\left(\mathscr{H}, d V_{\alpha}\right)$ to $L^{q}\left(\mathscr{H}, d V_{q \gamma+\alpha}\right)$.

(b) The operator $P_{\alpha}^{+}$is bounded from $L^{p}\left(\mathscr{H}, d V_{\alpha}\right)$ to $L^{q}\left(\mathscr{H}, d V_{q \gamma+\alpha}\right)$.

(c) The following relation holds:

$$
\frac{1}{p}-\frac{1}{q}=\frac{\gamma}{2+\alpha}
$$

For the proof of the sufficient part, we will use the offdiagonal Schur test due to Okikiolu [7].

\section{Proof of Theorem 1 and Proposition 2}

We start by recalling the following easy fact (see [3]).

Lemma 3. Let $\alpha$ be real. Then the function $f(z)=((z+$ it $) / i)^{-\alpha}$, with $t>0$, belongs to $L^{p}\left(\mathscr{H}, d V_{\nu}\right)$, if and only if $v>-1$ and $\alpha>(\nu+2) / p$. In this case,

$$
\|f\|_{p, \nu}^{p}=C_{\alpha, p, q} t^{-p \alpha+\nu+2} .
$$

The proof of the sufficient parts in our results is based on the following off-diagonal Schur-type test.

Lemma 4 (Okikiolu [7]). Let $p, r, q$ be positive numbers such that $1<p \leq r$ and $1 / p+1 / q=1$. Let $K(x, y)$ be a complexvalued function measurable on $X \times Y$ and suppose that there exist $0<t \leq 1$, measurable functions $\varphi_{1}: X \rightarrow(0, \infty)$ and $\varphi_{2}: Y \rightarrow(0, \infty)$, and nonnegative constants $M_{1}$ and $M_{2}$ such that

$$
\begin{gathered}
\int_{X}|K(x, y)|^{t q} \varphi_{1}^{q}(y) d \mu(y) \leq M_{1}^{q} \varphi_{2}^{q}(x) \quad \text { a.e on } Y, \\
\int_{Y}|K(x, y)|^{(1-t) r} \varphi_{2}^{r}(x) d \nu(x) \leq M_{2}^{r} \varphi_{1}^{r}(y) \text { a.e on } X .
\end{gathered}
$$

If $T$ is given by

$$
T f(x)=\int_{X} f(y) K(x, y) d \mu(y),
$$

where $f \in L^{p}(X, d \mu)$, then $T: L^{p}(X, d \mu) \rightarrow L^{r}(Y, d \nu)$ is bounded and for each $f \in L^{p}(X, d \mu)$,

$$
\|T f\|_{L^{r}(Y, d v)} \leq M_{1} M_{2}\|f\|_{L^{p}(X, d \mu)} .
$$

We prove the following.

Lemma 5. Let $\alpha>-1,0 \leq \gamma<2+\alpha$, and $1 \leq p \leq q<\infty$. If the operator $\mathscr{P}_{\alpha, \gamma}$ is bounded from $L^{p}\left(\mathscr{H}, d V_{\alpha}\right)$ to $L^{q}\left(\mathscr{H}, d V_{\alpha}\right)$, then

$$
\frac{1}{p}-\frac{1}{q}=\frac{\gamma}{2+\alpha}
$$

Proof. We assume that the operator $\mathscr{P}_{\alpha, \gamma}$ is bounded from $L^{p}\left(\mathscr{H}, d V_{\alpha}\right)$ to $L^{q}\left(\mathscr{H}, d V_{\alpha}\right)$. Let $R>0$ and associate to any function $f$, the function $f_{R}$ defined by $f_{R}(z)=f(R z)$. Then it is easy to see that

$$
\left\|f_{R}\right\|_{p, \alpha}=R^{-(2+\alpha) / p}\|f\|_{p, \alpha} .
$$

It follows also from an easy change of variables that

$$
\mathscr{P}_{\alpha, \gamma} f_{R}(w)=R^{-\gamma} \mathscr{P}_{\alpha} f(R w) .
$$

Hence

$$
\left\|\mathscr{P}_{\alpha, \gamma} f_{R}\right\|_{q, \alpha}=R^{-\gamma-(2+\alpha) / q}\left\|\mathscr{P}_{\alpha, \gamma} f\right\|_{q, \alpha} .
$$

It follows from the above considerations and the boundedness of $\mathscr{P}_{\alpha, \gamma}$ that there exists a constant $C>0$ such that, for any $f \in L^{p}\left(\mathscr{H}, d V_{\alpha}\right)$,

$$
\begin{aligned}
& R^{-\gamma-(2+\alpha) / q}\left\|\mathscr{P}_{\alpha, \gamma} f\right\|_{q, \alpha}=\left\|\mathscr{P}_{\alpha, \gamma} f_{R}\right\|_{q, \alpha} \leq C\left\|f_{R}\right\|_{p, \alpha} \\
& \quad=R^{-(2+\alpha) / p}\|f\|_{p, \alpha} .
\end{aligned}
$$

That is,

$$
R^{-\gamma-(2+\alpha) / q+(2+\alpha) / p}\left\|\mathscr{P}_{\alpha, \gamma} f\right\|_{q, \alpha} \leq C\|f\|_{p, \alpha} .
$$

As the latter holds for any $f \in L^{p}\left(\mathscr{H}, d V_{\alpha}\right)$ and any $R>0$, we must have

$$
-\gamma-\frac{2+\alpha}{q}+\frac{2+\alpha}{p}=0
$$

That is, $1 / p-1 / q=\gamma /(2+\alpha)$.

The following is obtained as above.

Lemma 6. Let $\alpha>-1,0 \leq \gamma<2+\alpha$, and $1 \leq p \leq q<\infty$. If the operator $P_{\alpha}$ is bounded from $L^{p}\left(\mathscr{H}, d V_{\alpha}\right)$ to $L^{q}\left(\mathscr{H}, d V_{q \gamma+\alpha}\right)$, then

$$
\frac{1}{p}-\frac{1}{q}=\frac{\gamma}{2+\alpha}
$$

We next prove that condition (5) is sufficient for the boundedness of the fractional operator in the case $p>1$.

Lemma 7. Let $\alpha>-1,0 \leq \gamma<2+\alpha$, and $1<p \leq q<\infty$. Assume that

$$
\frac{1}{p}-\frac{1}{q}=\frac{\gamma}{2+\alpha}
$$

Then the operator $\mathscr{P}_{\alpha, \gamma}^{+}$is bounded from $L^{p}\left(\mathscr{H}, d V_{\alpha}\right)$ to $L^{q}\left(\mathscr{H}, d V_{\alpha}\right)$.

Proof. We are assuming that $1 / p-1 / q=\gamma /(2+\alpha)$.

Let us put

$$
\omega=-(2+\alpha)\left(\frac{1}{p^{\prime}}+\frac{1}{q}\right)=-(2+\alpha)\left(1-\frac{\gamma}{2+\alpha}\right) .
$$


Clearly, $\omega<0$. As $\alpha+1>0$, we can find two numbers $s$ and $r$ such that

$$
\begin{aligned}
& 0<r<\frac{\alpha+1}{q}, \\
& 0<s<\frac{\alpha+1}{p^{\prime}}
\end{aligned}
$$

with $r>s$.

Let

$$
t:=\frac{-(\alpha+2) / p^{\prime}+s-r}{\omega}
$$

so that

$$
1-t=\frac{r-s-(\alpha+2) / q}{\omega} .
$$

We observe that the operator $\mathscr{P}_{\alpha, \gamma}^{+}$can be represented as

$$
\mathscr{P}_{\alpha, \gamma}^{+} f(z)=\int_{\mathscr{H}} f(w) K(z, w) d V_{\alpha}(w),
$$

where $K(z, w)=1 /|z-\bar{w}|^{2+\alpha-\gamma}$. Let us define

$$
\begin{aligned}
& \varphi_{1}(w)=(\Im w)^{-s}, \\
& \varphi_{2}(w)=(\Im w)^{-r} .
\end{aligned}
$$

Applying Okikiolu's test to $\mathscr{P}_{\alpha, \gamma}^{+}$we first obtain

$$
\begin{gathered}
\int_{\mathscr{H}} K(z, w)^{t p^{\prime}} \varphi_{1}^{p^{\prime}}(w)(\Im w)^{\alpha} d V(w) \\
=\int_{\mathscr{H}} \frac{v^{-s p^{\prime}+\alpha}}{|z-\bar{w}|^{t(2+\alpha-\gamma) p^{\prime}}} d V(w) .
\end{gathered}
$$

From our choice of $s$ we have $-s p^{\prime}+\alpha>-1$. Using the definitions of $\omega$ and $t$, we obtain

$$
\begin{aligned}
t( & +\alpha-\gamma) p^{\prime}+s p^{\prime}-\alpha-2 \\
& =(2+\alpha)\left(1-\frac{\gamma}{2+\alpha}\right) t p^{\prime}+s p^{\prime}-\alpha-2 \\
& =-\omega t p^{\prime}+s p^{\prime}-\alpha-2 \\
& =\left(\frac{\alpha+2}{p^{\prime}}+r-s\right) p^{\prime}+s p^{\prime}-\alpha-2=r p^{\prime}>0 .
\end{aligned}
$$

Hence we obtain from the above observations and Lemma 3 that

$$
\begin{aligned}
& \int_{\mathscr{H}} K(z, w)^{t p^{\prime}} \varphi_{1}^{p^{\prime}}(w)(\Im w)^{\alpha} d V(w)=C y^{-r p^{\prime}} \\
& =C \varphi_{2}(z)^{p^{\prime}} .
\end{aligned}
$$

In the same way, we first have

$$
\begin{gathered}
\int_{\mathscr{H}} K(z, w)^{(1-t) q} \varphi_{2}^{q}(z)(\Im z)^{\alpha} d V(z) \\
=\int_{\mathscr{H}} \frac{(\Im z)^{-r q+\alpha}}{|z-\bar{w}|^{(1-t)(2+\alpha-\gamma) q}} d V(z) .
\end{gathered}
$$

From our choice of $s$, we have $-r q+\alpha>-1$. From the definition of $\omega$ and $1-t$, we obtain

$$
\begin{aligned}
& (1-t)(2+\alpha-\gamma) q+r q-\alpha-2 \\
& \quad=-\omega(1-t) q+r q-\alpha-2 \\
& \quad=\left(\frac{2+\alpha}{q}+s-r\right) q+r q-\alpha-2=s q>0 .
\end{aligned}
$$

Hence we obtain from the above observations and Lemma 3 that

$$
\begin{aligned}
\int_{\mathscr{H}} K(z, w)^{(1-t) q} \varphi_{2}^{q}(z)(\Im z)^{\alpha} d V(w)=C(\Im w)^{-s q} \\
\quad=C \varphi_{1}(w)^{q} .
\end{aligned}
$$

The proof is complete.

Proof of Theorem 1. It is obvious that (b) $\Rightarrow$ (a). That (a) $\Rightarrow$ (c) is Lemma 5. That (c) $\Rightarrow$ (b) is Lemma 7. The proof is complete.

Proof of Proposition 2. Clearly, (b) $\Rightarrow$ (a). That (a) $\Rightarrow$ (c) is Lemma 6. That (c) $\Rightarrow$ (b) follows from Lemma 7 and the fact that the boundedness of $\mathscr{P}_{\alpha, \gamma}^{+}$from $L^{p}\left(\mathscr{H}, d V_{\alpha}\right)$ to $L^{q}\left(\mathscr{H}, d V_{\alpha}\right)$ implies the boundedness of $P_{\alpha}^{+}$from $L^{p}\left(\mathscr{H}, d V_{\alpha}\right)$ to $L^{q}\left(\mathscr{H}, d V_{q \gamma+\alpha}\right)$. The proof is complete.

\section{Conflicts of Interest}

The author declares that there are no conflicts of interest regarding the publication of this paper.

\section{References}

[1] B. Sehba, "Sharp weighted norm estimates for fractional Bergman operators," https://arxiv.org/abs/1703.00852.

[2] B. Sehba, "Sharp off-diagonal weighted norm estimates for the bergman projection," https://arxiv.org/abs/1703.00275.

[3] D. Békollé, A. Bonami, G. Garrigós, C. Nana, M. Peloso, and F. Ricci, "Lecture notes on Bergman projectors in tube domains over cones: an analytic and geometric viewpoint," in Proceedings of the International Workshop in Classical Analysis, (IMHOTEP '01), Yaoundé, Cameroon, 2001.

[4] E. M. Stein, Singular Integrals and Differentiability Properties of Functions, Princeton Mathematical Series, No. 30., Princeton University Press, Princeton, NJ, USA, 1970.

[5] R. Zhao, "Generalization of Schur's test and its application to a class of integral operators on the unit ball of $\mathrm{C}^{n}$," Integral Equations and Operator Theory, vol. 82, no. 4, pp. 519-532, 2015.

[6] J. S. Bansah and B. T. Sehba, "Boundedness of a family of Hilbert-type operators and its Bergman-type analogue," Illinois Journal of Mathematics, vol. 59, no. 4, pp. 949-977, 2015.

[7] G. O. Okikiolu, "On inequalities for integral operators," Glasgow Mathematical Journal, vol. 11, pp. 126-133, 1970. 


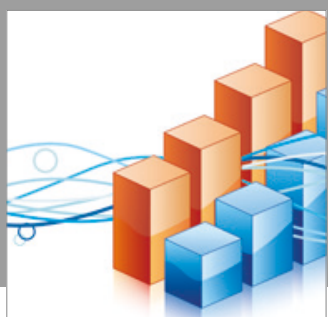

Advances in

Operations Research

vatersals

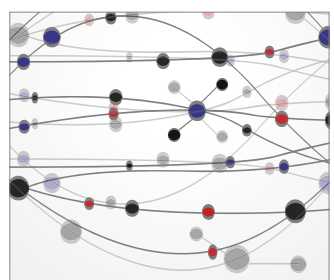

\section{The Scientific} World Journal
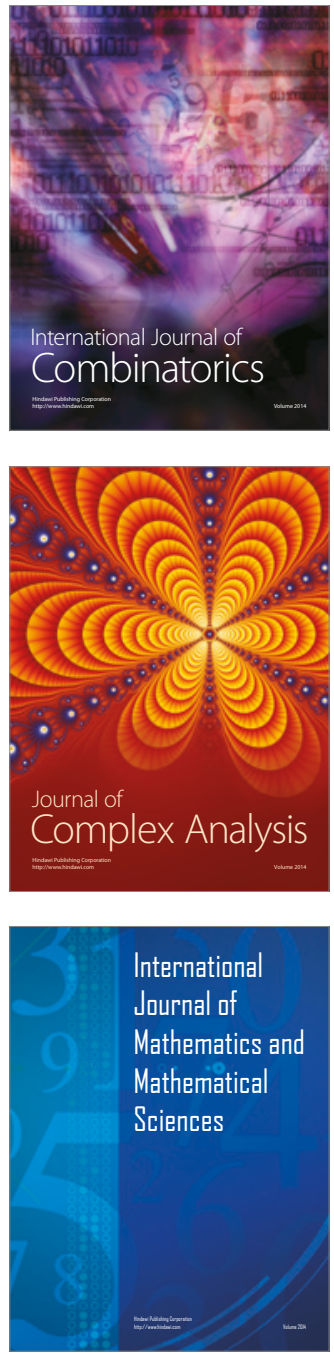
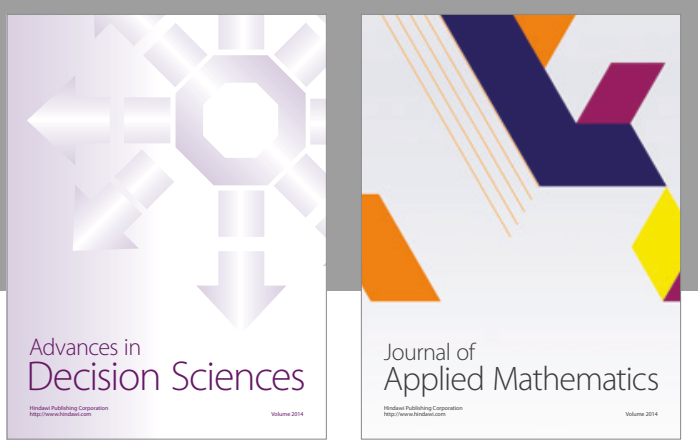

Algebra

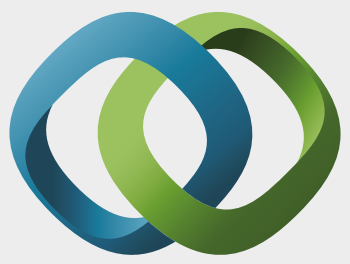

\section{Hindawi}

Submit your manuscripts at

https://www.hindawi.com
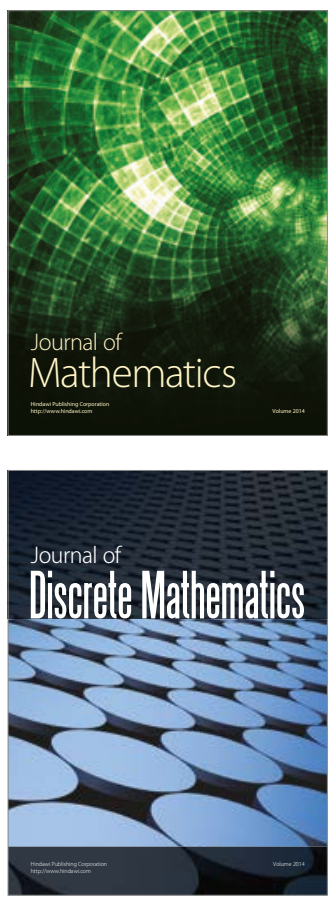

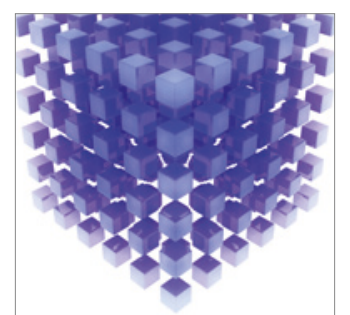

Mathematical Problems in Engineering
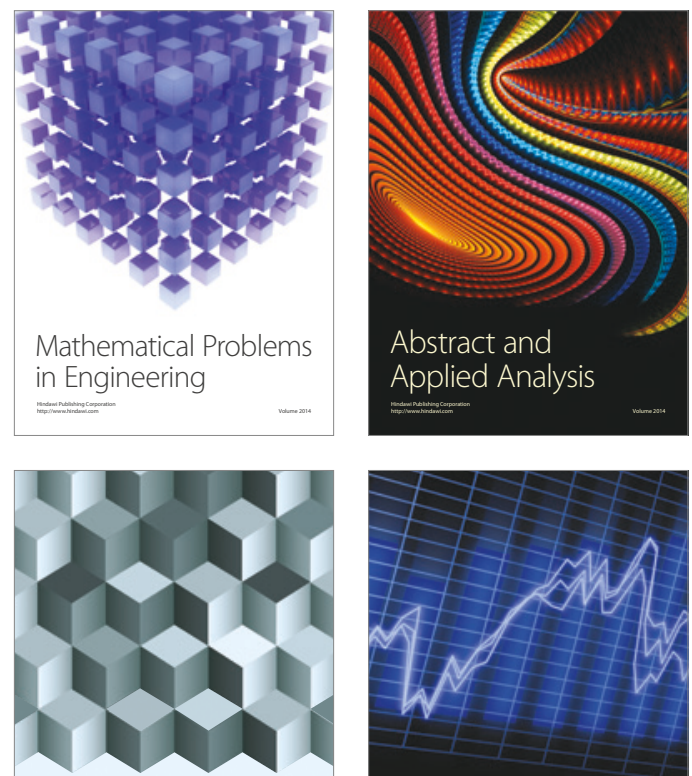

Journal of

Function Spaces

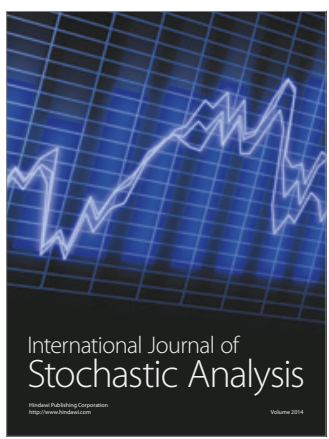

Probability and Statistics
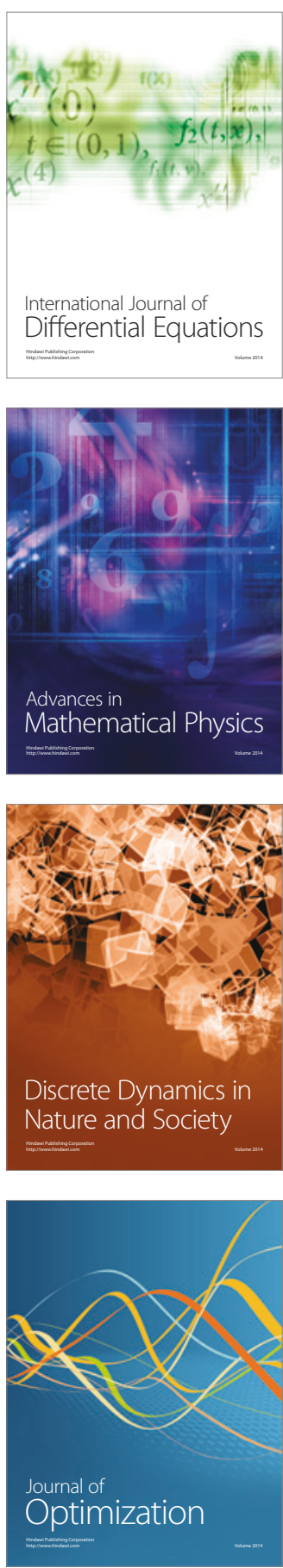